\title{
Beam-based measurements of persistent current decay in the Relativistic Heavy Ion Collider
}

\author{
W. Fischer, A. Jain, and S. Tepikian \\ Brookhaven National Laboratory, Upton, New York 11973 \\ (Received 26 December 2000; published 26 April 2001)
}

\begin{abstract}
The two rings of the Relativistic Heavy Ion Collider are equipped with superconducting dipole magnets. At injection, induced persistent currents in these magnets lead to a sextupole component. As the persistent currents decay with time, the horizontal and vertical chromaticities change. From magnet measurements of persistent current decays, chromaticity changes in the machine are estimated and compared with chromaticity measurements.
\end{abstract}

DOI: $10.1103 /$ PhysRevSTAB.4.041002

PACS numbers: 29.27.Fh, 85.25.Ly, 07.55.Ge, 73.23.Ra

\section{INTRODUCTION}

Persistent currents are eddy currents that are induced in the filaments of superconducting magnets through a change in the magnetic field. They lead to field distortions in the magnets. At a constant field during injection these currents decay with time. Decay rates typically vary from magnet to magnet and depend on the magnetic field history as well as on cable properties [1].

The magnetic field errors inside long straight magnets can be described through multipole coefficients according to

$$
B_{y}+i B_{x}=B_{\text {ref }} \sum_{n=0}^{\infty}\left(b_{n}+i a_{n}\right)\left(\frac{x+i y}{r_{0}}\right)^{n},
$$

where $B_{x}$ and $B_{y}$ denote the horizontal and vertical components of the magnetic field, respectively, $B_{\text {ref }}$ a reference field, $x$ and $y$ the horizontal and vertical position, $r_{0}$ a reference radius, and $i$ the imaginary unit. The reference radius chosen for most of the Relativistic Heavy Ion Collider (RHIC) magnets is $25 \mathrm{~mm}$. The multipole coefficients $b_{n}$ and $a_{n}$ denote the normal and skew components of the field, respectively, and are quoted in units of $10^{-4}$. We denote with $b_{0}$ a normal dipole. Persistent currents generate all multipoles which are allowed by coil symmetry, i.e., $b_{0}, b_{2}, b_{4}, \ldots$ in dipoles and $b_{1}, b_{5}, b_{9}, \ldots$ in quadrupoles.

At injection energy, the sextupole field errors $b_{2}$ from persistent currents can be significant due to their size and time dependence. The chromaticity stemming from persistent currents can surpass the natural chromaticity, and its uncorrected change during injection may be unacceptable for operation. Time dependence is not only observable as a slow decay at the injection level, but also as a snapback to the original multipole value when acceleration starts. For the four large hadron machines in existence or under construction, Table I gives an overview of the effect of persistent currents on the chromaticity at injection in various large hadron colliders [2-7]. For an effective correction of persistent current effects, reproducibility and predictability are important. In this article we compare, for both RHIC rings, the chromaticity changes expected from magnet measurements with measured chromaticity changes.

In the following, we present magnet measurements of persistent current decays, calculations of expected chromaticity changes at injection, and chromaticity measurements as a function of time. During the year 2000 gold run, beam was injected in RHIC at a dipole current of $462 \mathrm{~A}$ and ramped to $3169 \mathrm{~A}$ for physics stores. At the end of a store the dipole current was ramped down to a park level of $50 \mathrm{~A}$, after a stop at the injection level, and back to the injection level. The ramp rate between the injection and storage level was $25 \mathrm{~A} / \mathrm{s}$; it was $5 \mathrm{~A} / \mathrm{s}$ between the injection and park level. Time-dependent chromaticity measurements in both the blue and yellow rings started 2 min after reaching the injection current and extended over $15 \mathrm{~min}$.

TABLE I. Overview of the effect of persistent currents on the chromaticity at injection in various large hadron accelerators.

\begin{tabular}{lccccrrr}
\hline \hline & $\begin{array}{c}\text { Energy } \\
\text { range }\end{array}$ & \multicolumn{2}{c}{$\begin{array}{c}\text { Natural } \\
\text { chromaticity }\end{array}$} & \multicolumn{2}{c}{$\begin{array}{c}\text { Persistent current } \\
\text { chromaticity }\end{array}$} & \multicolumn{2}{c}{$\begin{array}{c}\text { Change over } \\
15 \text { min }^{\mathrm{a}}\end{array}$} \\
& $E_{\text {top }} / E_{\mathrm{inj}}$ & $\xi_{x, n}$ & $\xi_{y, n}$ & $\xi_{x, b_{2}}$ & $\xi_{y, b_{2}}$ & $\Delta \xi_{x}$ & $\Delta \xi_{y}$ \\
\hline Tevatron $^{\mathrm{b}}$ & 7 & -20 & -20 & -140 & +119 & +8 & -7 \\
HERA- $p$ & 23 & -44 & -47 & -275 & +245 & +13 & -11 \\
RHIC & 10 & -55 & -57 & -38 & +36 & +2 & -2 \\
LHC & 16 & -80 & -80 & -450 & +450 & +150 & -150 \\
\hline \hline
\end{tabular}

aUncorrected chromaticity change, starting 2 min after reaching the injection plateau.

${ }^{\mathrm{b}}$ Original fixed target configuration. 


\section{MAGNET MEASUREMENTS OF PERSISTENT CURRENT DECAYS}

The expected time-dependent chromaticity change can be deduced from two magnet measurements. First, the persistent current decay was measured in 20 magnets at $660 \mathrm{~A}$, a current higher than the injection current of $462 \mathrm{~A}$. Second, the decay was also measured in a single dipole magnet at $470 \mathrm{~A}$.

For the measurement of the decay in the 20 magnets, the current was ramped up to $5000 \mathrm{~A}$ and down to $25 \mathrm{~A}$ at a rate of $80 \mathrm{~A} / \mathrm{s}$, and then back up to $660 \mathrm{~A}$ at a rate of $16 \mathrm{~A} / \mathrm{s}$. Figure 1 shows the change in $b_{2}$ in these magnets after reaching $660 \mathrm{~A}$ on a linear time scale in part (a) and on a logarithmic time scale in part (b).

Ideally, the persistent current decay in magnets should be measured under the same conditions as in machine operation. However, the 20 RHIC magnets were measured during production assuming an injection current higher than the one finally used in operation. Later the injection energy was lowered to increase the injection kicker lifetime and to operate the machine at the same injection rigidity with gold and polarized protons. The maximum injection energy of the polarized protons is currently limited by the maximum beam energy for which polarization could be maintained during acceleration in the Alternating Gradient Synchrotron (AGS), which is the injector to RHIC. The injection energy is also limited by the spin-transparency of the transfer line to RHIC. The single magnet measurement was done during the commissioning at the injection current used in machine operation. In addition, during operation in 2000 the full current ramp rate was not yet achieved and thus the ramp rates in the magnet and beam-based measurements differ.

To describe the measured data, they were fitted to two functions. First, a logarithmic time dependence was assumed according to

$$
b_{2}=c_{0}+c_{1} \log _{10}\left(t / \tau_{1}\right) \quad \text { with } \tau_{1}=1 \mathrm{~s} .
$$

Such a time dependence is characteristic of a relaxation process, namely, the flux creep phenomenon in hard superconductors. The HERA magnets could be characterized this way [8]. Another fit for the RHIC magnets can be obtained with two superimposed exponentially decaying terms according to

$$
b_{2}=c_{0}+c_{1} e^{-t / \tau_{1}}+c_{2} e^{-t / \tau_{2}} .
$$

An exponential decay is expected when the eddy current loops are partly superconductive and partly resistive. Such loops could be between filaments or strands. Eddy current loops between filaments, however, should have decay constants of less than a second and should be irrelevant on the time scale of our investigation [8].

Since the time resolution of the measurement of the 20 magnets is only about $10 \mathrm{~s}$, the fitting was not extended below $10 \mathrm{~s}$. Furthermore, only two magnets were measured beyond $650 \mathrm{~s}$, and they do not appear to be in the middle of the distribution (see Fig. 1). The fit was therefore not extended beyond $650 \mathrm{~s}$. In Table II the fitted coefficients are shown for both functions together with the change in $b_{2}$ over 15 min, starting 2 min after reaching $660 \mathrm{~A}$. The $\Delta b_{2}$ errors are the square root of the average quadratic deviation between measured and fitted values. The fitted functions are also shown in Fig. 1. The fit with Eq. (2) overestimates the decay while the fit (3) underestimates the decay.

Since the persistent currents are approximately independent of the main field at transport currents well below the critical current, the coefficients $c_{0}, c_{1}$, and $c_{2}$ in Eqs. (2) and (3) may be scaled with the reference field. For $462 \mathrm{~A}$ the average dipole field is $0.3260 \mathrm{~T}$; for $660 \mathrm{~A}$ the field is $0.4663 \mathrm{~T}$. The result of scaling is also shown in Table II.

The simple scaling is, however, only approximate, and the exact scaling is not known. Measurements in a single LHC dipole prototype, built at Brookhaven, seem to indicate that such a simple scaling tends to overestimate the persistent current decay when scaled from higher to lower current. Furthermore, the magnets were cycled to 5000 A,

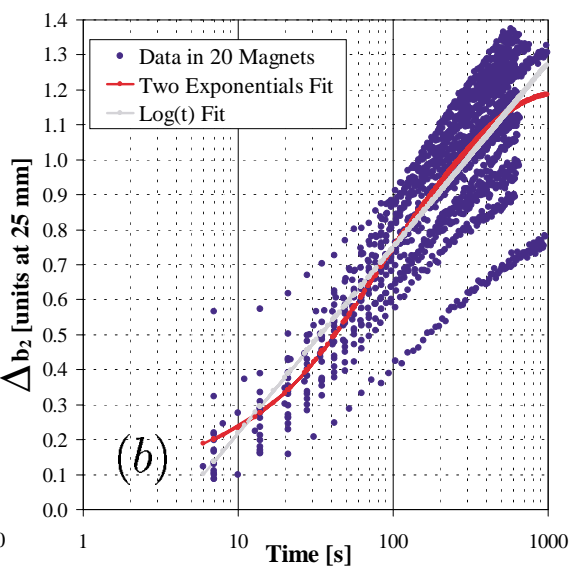

FIG. 1. (Color) Time-dependent change of the sextupole coefficient in 20 RHIC dipoles at 660 A. Part (a) shows the dependence on a linear time scale, part (b) on a logarithmic $\left(\log _{10}\right)$ time scale. 
TABLE II. Time-decay coefficients from magnet measurements. Coefficients for the logarithmic fit are according to Eq. (2), and coefficients for the two exponentials fit are according to Eq. (3). The change in $b_{2}$ is computed from 2 to 17 min with the fitted functions. The $\Delta b_{2}$ errors are the square root of the average quadratic deviation between measured and fitted values.

\begin{tabular}{cccccccc}
\hline \hline & \multicolumn{3}{c}{ Logarithmic fit } & \multicolumn{4}{c}{ Two exponentials fit } \\
Measurement & $c_{1}$ & $\Delta b_{2}$ & $c_{1}$ & $\tau_{1}$ & $c_{2}$ & $\tau_{2}$ & $\Delta b_{2}$ \\
& $\left(10^{-4}\right)$ & $\left(10^{-4}\right)$ & $\left(10^{-4}\right)$ & $(\mathrm{s})$ & $\left(10^{-4}\right)$ & $(\mathrm{s})$ & $\left(10^{-4}\right)$ \\
\hline 20 magnets at 660 A, average & +0.53 & $0.49 \pm 0.15$ & -0.49 & 47 & -0.59 & 241 & $0.39 \pm 0.14$ \\
20 magnets scaled to 462 A & +0.76 & $0.70 \pm 0.22$ & -0.70 & 47 & -0.84 & 241 & $0.56 \pm 0.20$ \\
1 magnet at 470 A & +0.89 & $0.83 \pm 0.07$ & -1.40 & 45 & -0.45 & 303 & $0.39 \pm 0.02$ \\
\hline \hline
\end{tabular}

higher than the 3169 A used in the beam-based measurements. Measurements in a single RHIC dipole show that the persistent currents decay faster when the magnet is cycled to a higher current [9]. In Ref. [10] it was found that the effect of the operational cycle on the snapback could be modeled much better with a neural network than with a simple phenomenological model. Some information on scaling may also be derived from the work in Ref. [11].

Extensive measurements of dynamic effects, including the sextupole time decay and snapback, have been carried out with a single dipole using a fast measuring coil system that rotates with a period of $0.66 \mathrm{~s}$. A detailed report summarizes these data [9].

Figure 2 shows the sextupole time decay at $470 \mathrm{~A}$, after cycling the magnet to $3500 \mathrm{~A}$ and down to $25 \mathrm{~A}$ before bringing it to the injection level. The ramp rate during the cycling was $60 \mathrm{~A} / \mathrm{s}$. The time $t=0$ is chosen to be the moment when the power supply current reaches 450 A. Part (a) shows $b_{2}$ on linear time scale, part (b) on a logarithmic time scale. It is evident that a simple linear relation between $b_{2}$ and $\log (t)$ is not followed by the data over the entire range. The data were also fitted to the functions (2) and (3). In the fits, data points below $7 \mathrm{~s}$ were disregarded since they would contribute a much larger error to the fit parameters than the other data points. The fitting results are shown in Table II.

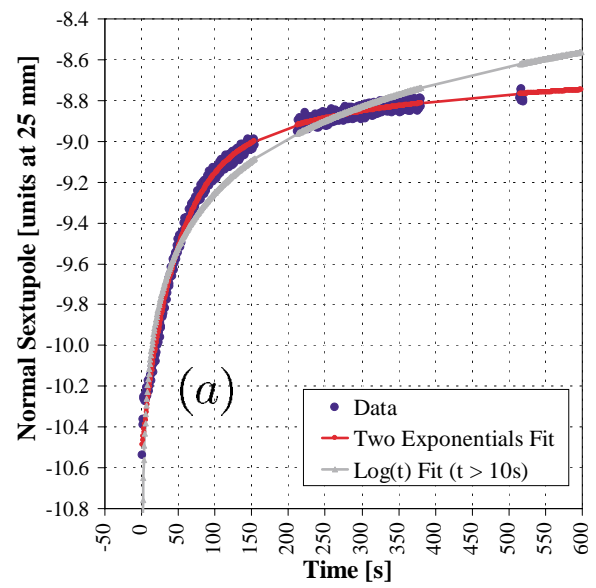

FIG. 2. (Color) Time-dependent sextupole coefficient in a single RHIC dipole at 470 A. Part (a) shows the dependence on a linear time scale, part (b) on a logarithmic $\left(\log _{10}\right)$ time scale.

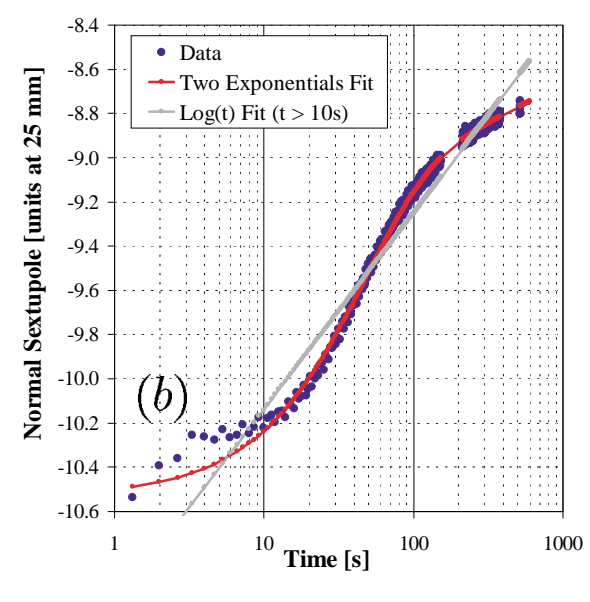

For beam-based measurements, a time interval of $15 \mathrm{~min}$, starting at $2 \mathrm{~min}$ after reaching the injection level, was covered. This lies partially outside the time interval of the magnet measurements. However, the changes beyond $600 \mathrm{~s}$ are small. Based on the available data and the fits given by Eqs. (2) and (3), $b_{2}$ should not change by more than 0.15 units between 600 and $1020 \mathrm{~s}$.

The expected change in $b_{2}$ from the scaled measurements of the 20 magnets at 660 A differs from the expected change in $b_{2}$ from the single magnet measurement at $470 \mathrm{~A}$ by some $25 \%$, with error intervals overlapping (see Table II). However, as Fig. 1 shows, individual magnets can differ significantly in their behavior from each other. The change in the sextupole coefficient from 150 to $600 \mathrm{~s}$ ranged from 0.15 to 0.36 units, with a mean of 0.28 units and a standard deviation of 0.05 units.

The dependence of the persistent current decay on the ramp rate has been measured in a LHC prototype magnet. This magnet uses the same coil design as the RHIC dipoles, but is a twin aperture magnet with a different yoke design. The superconductor is also different from the RHIC conductor. For the LHC prototype, the difference in the fit parameters $c_{0}, c_{1}, c_{2}$, and $\tau_{1}, \tau_{2}$ in Eqs. (2) and (3) for ramp rates of $25 \mathrm{~A} / \mathrm{s}$ and $50 \mathrm{~A} / \mathrm{s}$ does not exceed $9 \%$. We therefore disregard this effect. 
Magnet measurements of persistent current decays for the Tevatron are reported in Ref. [2], for HERA in Refs. [12,13], and for the LHC in Refs. [14-16].

\section{EXPECTED CHROMATICITY CHANGE FROM PERSISTENT CURRENT DECAY}

In this section we compute the expected chromaticity change from the magnet measurements shown in Sec. II. We will make an analytical estimate and use two optics programs for this purpose. An overview of RHIC is given in Ref. [17].

\section{A. Analytical estimate}

We use as definition for the chromaticity

$$
\xi_{x, y}=\frac{\Delta Q_{x, y}}{\Delta p / p},
$$

where $Q_{x, y}$ are the transverse tunes and $\Delta p / p$ is the relative momentum deviation. The change of chromaticity $\Delta \xi_{x, y}$ associated with the change of the sextupole component $\Delta b_{2}$ can be computed as (see, for example, Refs. [18,19])

$$
\Delta \xi_{x, y}= \pm \frac{1}{2 \pi} \frac{1}{(B \rho)} \oint \beta_{x, y}(s) \frac{B_{\mathrm{ref}} \Delta b_{2}(s)}{r_{0}^{2}} \eta_{x}(s) d s,
$$

where $\beta_{x, y}$ is the lattice beta function and $\eta_{x}$ the dispersion. $B_{\text {ref }}$ may be different from the $B$ in the rigidity $(B \rho)$. Assuming sextupole errors only in dipoles Eq. (5) can be approximated as

$$
\Delta \xi_{x, y}= \pm \frac{1}{2 \pi} \frac{1}{(B \rho)} \frac{B_{\mathrm{ref}} \Delta b_{2}}{r_{0}^{2}} N I_{x, y},
$$

where $N$ denotes the number of dipoles and the integrals

$$
I_{x, y}=\int_{l_{1}}^{l_{2}} \beta_{x, y}(s) \eta_{x}(s) d s
$$

extend over a dipole in a FODO cell. In Eq. (6) we use only the average change in the sextupole coefficient $\Delta b_{2}$. Furthermore, we consider only dipoles in FODO cells. With optics programs it can be determined that the contribution from the interaction regions is only $\Delta \xi_{x, y}=$ $0.1 \Delta b_{2, \mathrm{IR}}$, which is small compared with the whole machine (see Table I). This is due to the fact that the DX (beam separators) and D0 magnets on both sides of an interaction region almost cancel each other, and the dispersion in the remaining interaction region dipoles is small.

For simplicity we assume that the FODO cells of length $L_{p}$ have equal phase advance $\mu$ in both planes and a bending angle $\phi$ for the whole cell. The integrals $I_{x, y}$ can be computed using a thin-lens approximation as [20]

$$
I_{x}=\int_{l_{1}}^{l_{2}} d s\left[\beta^{+}-2 s \frac{1+\sin \frac{\mu}{2}}{\cos \frac{\mu}{2}}+4 s^{2} \frac{\tan \frac{\mu}{2}}{L_{p}}\right]\left[\eta^{+}\left(1-2 s \frac{\sin \frac{\mu}{2}}{L_{p}}\right)+s^{2} \frac{\phi}{2 L_{p}}\right]
$$

and

$$
I_{y}=\int_{l_{1}}^{l_{2}} d s\left[\beta^{+}-2\left(\frac{L_{p}}{2}-s\right) \frac{1+\sin \frac{\mu}{2}}{\cos \frac{\mu}{2}}+4\left(\frac{L_{p}}{2}-s\right)^{2} \frac{\tan \frac{\mu}{2}}{L_{p}}\right]\left[\eta^{+}\left(1-2 s \frac{\sin \frac{\mu}{2}}{L_{p}}\right)+s^{2} \frac{\phi}{2 L_{p}}\right] .
$$

The maximum beta function $\beta^{+}$and dispersion $\eta^{+}$of the cell are given by [20]

$$
\beta^{+}=\frac{L_{p}\left(1+\sin \frac{\mu}{2}\right)}{\sin \mu} \text { and } \eta^{+}=\frac{L_{p} \phi\left(1+\frac{1}{2} \sin \frac{\mu}{2}\right)}{4 \sin ^{2} \frac{\mu}{2}} .
$$

For RHIC at injection we have $(B \rho)=79.0 \mathrm{Tm}, B_{\text {ref }}=$ $0.326 \mathrm{~T}, r_{0}=25 \mathrm{~mm}, N=144, l_{1}=2.5 \mathrm{~m}, l_{2}=12 \mathrm{~m}$, $L_{p}=29.6 \mathrm{~m}, \mu=1.41 \mathrm{rad}$, and $\phi=77.8 \mathrm{mrad}$. Substituting everything in Eq. (6) and solving the integrals $I_{x, y}$ numerically we obtain

$$
\Delta \xi_{x}=4.9 \Delta b_{2} \quad \text { and } \quad \Delta \xi_{y}=-4.4 \Delta b_{2} .
$$

\section{B. Estimate from optics programs}

For RHIC we use two optics programs: MAD [21] for design and TEAPOT [22] as an on-line model in operation. Both programs can introduce magnetic field errors in the lattice. The natural chromaticity and the effect of sextupole field errors in the dipoles were determined and are shown in Table III together with the analytical estimate.
The analytical estimate and the coefficients determined from MAD agree very well. The TEAPOT coefficients are slightly different since TEAPOT uses a single kick approximation for every dipole, while the analytical estimate and MAD use a thick lens model. An estimate for the expected difference between MAD and TEAPOT can be obtained by replacing the integrals $I_{x, y}$ in Eq. (6) by the product $\beta_{x, y}\left(\frac{l_{1}+l_{2}}{2}\right) \eta_{x}\left(\frac{l_{1}+l_{2}}{2}\right)\left(l_{2}-l_{1}\right)$, which uses only the function values in the center of the dipole. A difference of $0.4 \Delta b_{2}$ is obtained, which explains most of the difference between the MAD and TEAPOT coefficients.

TABLE III. The effect of a change in the sextupole component on the chromaticity from an analytical estimate, MAD and TEAPOT. $b_{2}$ is given in units of $10^{-4}$ at a reference radius of $r_{0}=25 \mathrm{~mm}$.

\begin{tabular}{lcccc}
\hline \hline & \multicolumn{2}{c}{ Natural } & \multicolumn{2}{c}{ Chromaticity due } \\
& \multicolumn{2}{c}{ chromaticity } & \multicolumn{2}{c}{ to $b_{2}$ in dipoles } \\
& $\xi_{x, n}$ & $\xi_{y, n}$ & $\xi_{x, b_{2}}$ & $\xi_{y, b_{2}}$ \\
\hline Analytical & $\ldots$ & $\ldots$ & $+4.9 b_{2}$ & $-4.4 b_{2}$ \\
MAD & -54.9 & -56.5 & $+4.9 b_{2}$ & $-4.3 b_{2}$ \\
TEAPOT & -54.7 & -56.6 & $+4.2 b_{2}$ & $-4.0 b_{2}$ \\
\hline \hline
\end{tabular}




\section{MEASUREMENTS OF THE TIME-DEPENDENT CHROMATICITY}

The chromaticity was determined by measuring the tune at different average radii and, therefore, momenta. The tune was obtained from the Fourier transforms of transverse beam oscillations over 512 turns after the beam was excited with a single small kick [23]. The momentum was changed by $\Delta p / p= \pm 0.0017$. The chromaticity was measured every $16 \mathrm{~s}$ beginning $2 \mathrm{~min}$ after reaching the injection plateau. Measurements extended over 15 min. Both the blue and the yellow ring were measured in gold operation. In Figs. 3 and 4 the results of the timedependent chromaticity measurements are shown on a linear and logarithmic time scale, respectively.

With a partially squeezed optic in RHIC, secondorder chromaticity changes may be important. Secondand third-order chromaticity coefficients were computed with MAD's HARMON module. With momentum changes of $\Delta p / p= \pm 0.0017$, tune changes from second- and third-order chromaticity do not exceed 0.001 , which is the resolution of the tune measurement. Second-order chromaticity changes could therefore not be measured with this optic.

Since the chromaticity change is proportional to the change in the sextupole coefficient $b_{2}$ [see Eq. (6)], we attempt to fit the chromaticity to two functions equivalent to Eqs. (2) and (3). These are

$$
\xi_{x, y}=\bar{c}_{0}+\bar{c}_{1} \log _{10}\left(t / \bar{\tau}_{1}\right) \text { with } \bar{\tau}_{1}=1 \mathrm{~s},
$$

and

$$
\xi_{x, y}=\bar{c}_{0}+\bar{c}_{1} e^{-t / \bar{\tau}_{1}}+\bar{c}_{2} e^{-t / \bar{\tau}_{2}}
$$

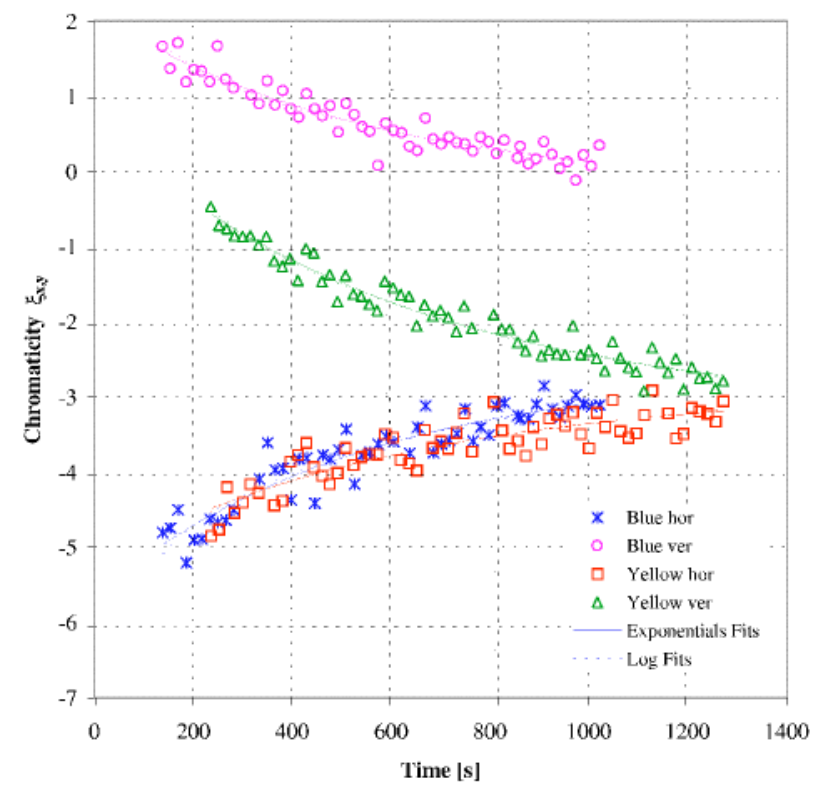

FIG. 3. (Color) Measured chromaticity in the two RHIC rings on a linear time scale.
The experimental data make a fit to five parameters in Eq. (13) difficult. Furthermore, the fast decaying component in Eq. (13) has largely decayed when the beam-based measurements start (compare with Table II). We therefore set $\bar{c}_{1}=0$ and perform only a three parameter fit. In addition, we force the time constant $\bar{\tau}_{2}$ to be the same in the blue and yellow ring fit since the number of data points is limited. A small number of data points results in a large error of the fit parameter. According to Eq. (6) the time constant should be the same for both rings and plans.

The results are shown in Table IV along with the change in chromaticity over a 15 min interval starting 2 min after reaching the injection level. The $\Delta \xi_{x, y}$ errors correspond to the square root of the average quadratic deviation between measured and fitted values.

Table IV also shows the chromaticity change expected from both magnet measurements, the scaled measurement of the 20 magnets and the single magnet measurement. The beam-based measurements agree better with the single magnet measurement than with the scaled measurement of the 20 magnets. The coefficients $\kappa$ in Table IV give the deviation of the chromaticity measurement to the expectation from the magnet measurement. Generally, a better agreement is obtained for the exponential fits. For these, the scaled 660 A measurements $\kappa_{660} \mathrm{~A}$ range from $-27 \%$ to $0 \%$ with an average of $-28 \%$. For the single $470 \mathrm{~A}$ measurement, $\kappa_{470}$ A ranges from $-22 \%$ to $44 \%$ with an average of $4 \%$. The single magnet is a relatively good representative for the machine.

Figure 5 also shows the comparison between the expectation from both magnet measurements and the chromaticity measurements, again on a linear and a logarithmic time scale. The single magnet behavior not only agrees much

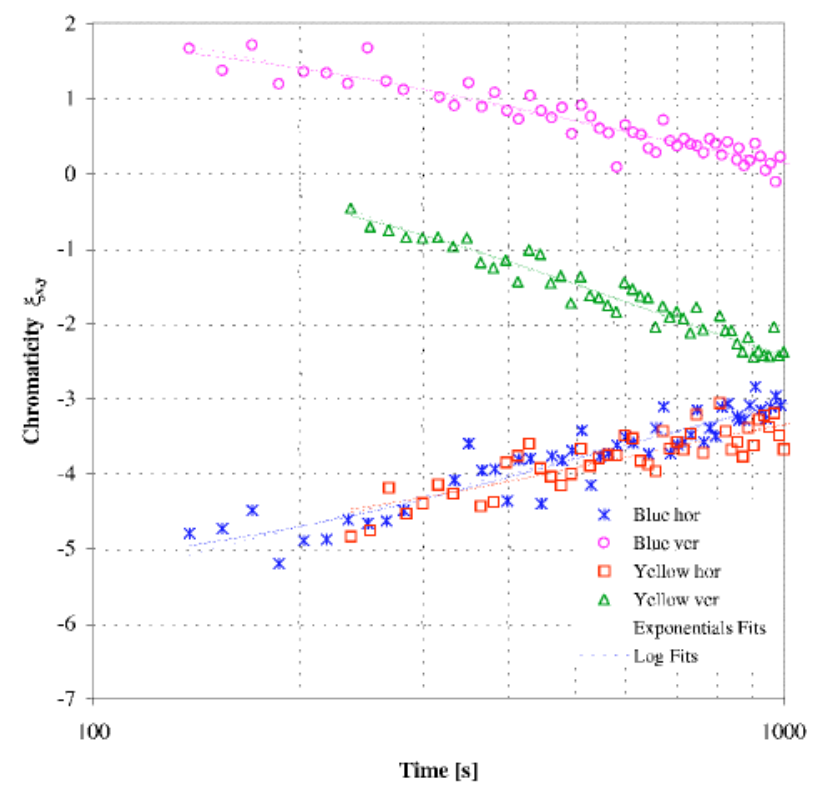

FIG. 4. (Color) Measured chromaticity in the two RHIC rings on a logarithmic $\left(\log _{10}\right)$ time scale. 
TABLE IV. Chromaticity time-decay coefficients from magnet and beam-based measurements. Coefficients for the logarithmic fit are according to Eq. (12), and coefficients for the two exponentials fit are according to Eq. (13). The change in $\xi_{x, y}$ is computed from 2 to $17 \mathrm{~min}$ with the fitted functions. $\kappa$ denotes the difference in the chromaticity change between the beam-based measurements and the expectations from the magnet measurements. The subscript of $\kappa$ denotes the magnet measurement used for the comparison. The $\Delta \xi_{x, y}$ errors correspond to the square root of the average quadratic deviation between measured and fitted values.

\begin{tabular}{|c|c|c|c|c|c|c|c|c|c|c|c|}
\hline \multirow[b]{2}{*}{ Measurement } & \multicolumn{4}{|c|}{ Logarithmic fit } & \multicolumn{7}{|c|}{ Exponentials fit } \\
\hline & $\begin{array}{c}\bar{c}_{1} \\
\left(10^{-4}\right)\end{array}$ & $\begin{array}{l}\Delta \xi_{x, y} \\
\text { (1) }\end{array}$ & $\begin{array}{c}\kappa_{660 \mathrm{~A}} \\
(\%)\end{array}$ & $\begin{array}{c}\kappa_{470 \mathrm{~A}} \\
(\%)\end{array}$ & $\begin{array}{c}\bar{c}_{1} \\
\left(10^{-4}\right)\end{array}$ & $\begin{array}{l}\bar{\tau}_{1} \\
(\mathrm{~s})\end{array}$ & $\begin{array}{c}\bar{c}_{2} \\
\left(10^{-4}\right)\end{array}$ & $\begin{array}{l}\bar{\tau}_{2} \\
(\mathrm{~s})\end{array}$ & $\begin{array}{c}\Delta \xi_{x, y} \\
(1)\end{array}$ & $\begin{array}{c}\kappa_{660 \mathrm{~A}} \\
(\%)\end{array}$ & $\begin{array}{c}\kappa_{470 \mathrm{~A}} \\
(\%)\end{array}$ \\
\hline \multicolumn{12}{|l|}{ Horizontal } \\
\hline From 20 magnets, 660 A scaled & +3.71 & $3.45 \pm 1.06$ & & & -3.43 & 47 & -4.14 & 241 & $2.73 \pm 0.97$ & & \\
\hline From single magnet, $470 \mathrm{~A}$ & +4.36 & $4.05 \pm 0.34$ & & & -6.86 & 45 & -2.21 & 303 & $1.89 \pm 0.10$ & & \\
\hline Blue ring & +2.32 & $2.16 \pm 0.22$ & -38 & -47 & $\ldots$ & $\ldots$ & -3.04 & 531 & $1.98 \pm 0.21$ & -27 & +5 \\
\hline Yellow ring & +1.86 & $1.73 \pm 0.20$ & -50 & -57 & $\cdots$ & $\cdots$ & -2.34 & 623 & $1.48 \pm 0.21$ & -46 & -22 \\
\hline \multicolumn{12}{|l|}{ Vertical } \\
\hline From 20 magnets, 660 A scaled & -3.34 & $-3.10 \pm 0.95$ & & & 3.08 & 47 & 3.71 & 241 & $-2.45 \pm 0.87$ & & \\
\hline From single magnet, $470 \mathrm{~A}$ & -3.92 & $-3.64 \pm 0.31$ & & & 6.16 & 45 & 1.98 & 303 & $-1.70 \pm 0.09$ & & \\
\hline Blue ring & -1.81 & $-1.68 \pm 0.16$ & -46 & -54 & $\ldots$ & $\ldots$ & 2.37 & 531 & $-1.54 \pm 0.15$ & -37 & -9 \\
\hline Yellow ring & -3.07 & $-2.85 \pm 0.15$ & -8 & -22 & $\cdots$ & $\ldots$ & 3.87 & 623 & $-2.44 \pm 0.15$ & \pm 0 & +44 \\
\hline
\end{tabular}
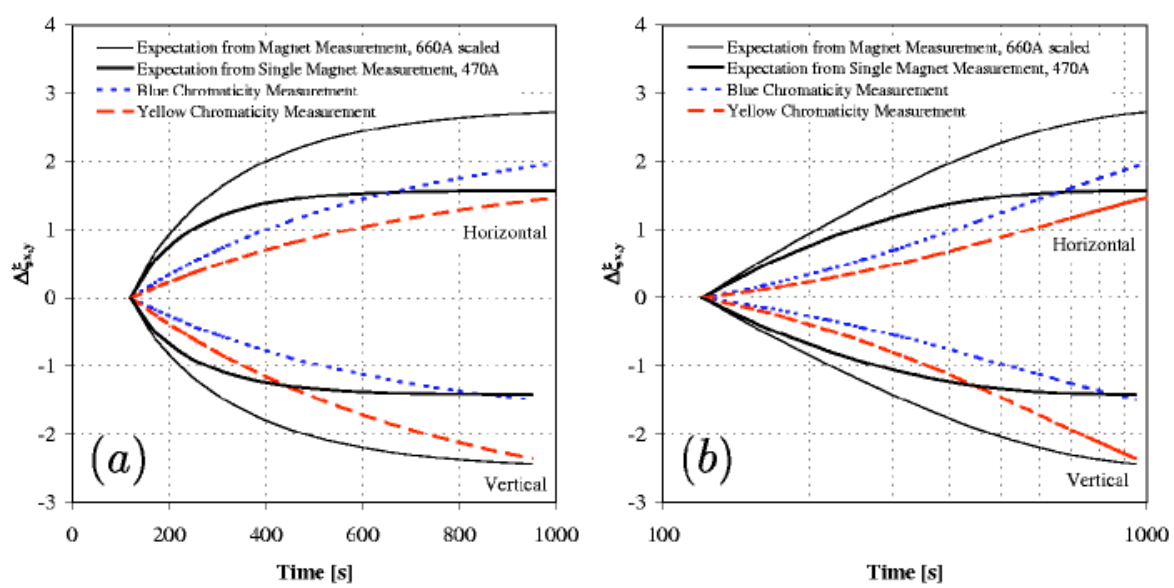

FIG. 5. (Color) Comparison between chromaticity changes expected from magnet measurements and measured chromaticity changes on a linear time scale in part (a) and logarithmic $\left(\log _{10}\right)$ time scale in part (b). In all cases the exponential fit functions are shown.

better with the chromaticity measurements at the end of the measurement interval, but also during the whole interval.

\section{SUMMARY}

Magnet measurements of persistent current decays in RHIC dipoles show neither a clear logarithmic nor a clear exponential dependence but indicate both components. The relatively small chromaticity changes that can be computed from the measurements of a single magnet agree well with beam-based measurements.

A single magnet was found to be a good proxy for both the blue and the yellow ring with respect to the persistent current decay at injection. However, since decay times vary considerably from magnet to magnet, predictions of the chromaticity change should not rely on measurements of a single magnet.
The agreement with a scaled measurement of 20 magnets, carried out at a higher current and with a different cycle, is less satisfactory. This suggests that the simple scaling law used is only a crude approximation. For machines, for which a detailed knowledge of the persistent current behavior is essential for operation, magnet measurements should therefore be done under conditions as close as possible to those encountered in machine operation.

\section{ACKNOWLEDGMENTS}

We are thankful to R. Thomas and W. Louie for help with the magnet measurements and to the RHIC operations teams for help during the beam-based measurements. We are grateful to A. Drees for support of the tune measurement systems. A special thanks to T. Satogata and J. van Zeijts who supported this study in many ways. 
[1] P. Schmüser, in Handbook of Accelerator Physics and Engineering, edited by A. W. Chao and M. Tigner (World Scientific, Singapore, 1999).

[2] D. A. Herrup, W. Kinney, M. J. Lamm, and A. Mokhtarani, Phys. Rev. E 49, 5660-5567 (1994).

[3] D. Herrup (private communication).

[4] B. J. Holzer, Part. Accel. 55, 461-471 (1996); 55, 215-225 (1996).

[5] C. Montag and B. Holzer, in Proceedings of the US-LHC Collaboration Meeting on Accelerator Physics Experiments for Future Hadron Colliders (BNL Formal Report No. 52601, 2000).

[6] O. Brüning, in Proceedings of Chamonix X, Chamonix, France (CERN Report No. CERN-SL-2000-007 DI, 2000).

[7] L. Bottura, in Proceedings of the VLHC Annual Meeting, Port Jefferson, New York, 2000 (http://magnets.rhic. bnl.gov/vlhc101600/Presentations/Luca_Bottura.pdf).

[8] K.-H. Mess and P. Schmüser, in Proceedings of the CERN Accelerator School on Superconductivity in Particle Accelerators, Hamburg, Germany, 1988 (CERN Report No. CERN 89-04, 1989).

[9] A. Jain, G. Ganetis, J. Muratore, R. Thomas, and P. Wanderer, BNL Magnet Division Note 593-11 (AM-MD-294), 2000; http://magnets.rhic.bnl.gov/publications/documents/ FastMeasurements.pdf

[10] M. Schneider, Ph.D. thesis, Technische Universität Vienna, Austria, 2000.

[11] C. Völlinger, M. Aleksa, and S. Russenschuck, Phys. Rev. ST Accel. Beams 3, 122402 (2000).

[12] H. Brück, D. Gall, G. Knies, J. Krzywinski, R. Lange, R. Meinke, H. Preissner, P. Schmüser, and Y. Zhao, in Proceedings of the 1990 European Particle Accelerator Conference, Nice, France (Editions Frontíeres, Gif-sur-Yvette,
France, 1990).

[13] H. Brück, D. Gall, J. Krzywinski, R. Lange, R. Meinke, H. Preissner, P. Schmüser, and Y. Zhao, in Proceedings of the 1990 European Particle Accelerator Conference, Nice, France (Ref. [12]).

[14] L. Bottura, L. Walckiers, and R. Wolf, IEEE Trans. Appl. Supercond. 7, 602-605 (1997).

[15] M. Haverkamp, L. Bottura, and M. Schneider, in Proceedings of the 4th European Conference on Applied Superconductivity, Sitges, Spain, 1999 (Institute of Physics, Bristol, UK, 2000).

[16] M. Haverkamp, A. Kuijper, A. den Ouden, B. ten Haken, L. Bottura, and H. H.J. ten Kate, in Proceedings of the Conference on Applied Superconductivity, Virginia Beach, Virginia, 2000 (IEEE, Piscataway, NJ, 2000).

[17] The RHIC Design Manual, edited by H. Hahn, Revised October, 2000 (http://www.rhichome.bnl.gov/NT-share/ rhicdm/00_toc1i.htm).

[18] M. Conte and W. W. MacKay, An Introduction to the Physics of Particle Accelerators (World Scientific, Singapore, 1991).

[19] D. A. Edwards and M. Syphers, in Handbook of Accelerator Physics and Engineering (Ref. [1]).

[20] E. Keil, in Handbook of Accelerator Physics and Engineering (Ref. [1]).

[21] H. Grote and F. C. Iselin, CERN Report No. CERN/ SL/90-13 (AP) Rev. 5, 1996.

[22] F. Pilat, S. Tepikian, and C. Trahern, Report No. RHIC/ AP/146, 1998.

[23] P. Cameron, R. Connolly, A. Drees, T. Ryan, H. Schmickler, T. Shea, and D. Trbojevic, BNL Report No. RHIC/ AP/156, 1998. 